\title{
Renal Infiltration of Follicular Lymphoma
}

\author{
Folliküler Lenfomada Renal Infiltrasyon
}

\author{
Ivan Petković1, Miljan Krstić2,3, Ivica Pejcić1,3, Svetislav Vrbić1,3, Slavica Stojnev2,3, Ana Cvetanović1, \\ Mirjana Balićl, Mirjana Todorović ${ }^{1}$ \\ ${ }^{1}$ Niš University Clinical Center, Clinic of Oncology, Department of Hematological Malignancies, Niš, Serbia \\ 2Niš University Clinical Center, Institute of Pathology, Niš, Serbia \\ ${ }^{3}$ Niš University Faculty of Medicine, Niš, Serbia
}

To the Editor,

We present a case of renal infiltration of grade $3 \mathrm{~A}$ follicular lymphoma (FL) mimicking renal cell carcinoma (RCC).

Chronic renal failure (CRF) was diagnosed in a 63-yearold male during routine health controls. Ultrasonography results emphasized a left renal mass. Multislice computed tomography (MSCT) confirmed the prior findings of a solid multilobular mass $(48 \times 46 \times 79 \mathrm{~mm})$, reduced kidney parenchyma, and intact surrounding lymph nodes (LNs) mimicking RCC. Without previous oncological evaluation, urologists performed nephrectomy. Histopathology revealed FL of grade $3 \mathrm{~A}$, with perirenal infiltration. Immunohistochemistry was typical: CD79 $\alpha+$, CD20+, CD10+, MUM1-, Bcl-2+, Bcl-6+, cyclin D1-, CD23-, CD3, CD5-, CK AE1/AE3-, and EMA-, with a Ki-67 index of $20 \%$ (Figure 1). After surgery, the patient underwent a lymphoma staging procedure at our clinic. MSCT scans, bone marrow biopsy, and blood analysis results were within normal ranges. Only residual CRF was maintained and $\beta 2$ microglobulin level was $6.2 \mathrm{mg} / \mathrm{L}$. The performed PET/ CT detected left-sided paravertebral (Th4, Th5) and axilla LNs [standardized uptake value (SUV) max: 7.4]. Since it was high-grade advanced FL with a Follicular International Prognostic Index (FLIPI) score of 2, we conducted 8 R-CHOP induction cycles (left ventricular ejection fraction: 70\%). Posttreatment PET/CT verified complete response. Informed consent was obtained from the patient.
Primary renal lymphoma (PRL) is defined as lymphoma arising in the renal parenchyma and not invasion from an adjacent lymphomatous mass [1]. Since the kidney is not a lymphoid organ, proposed mechanisms may include dissemination from subcapsular lymphatics, retroperitoneal lymphoma extension, or lymphoplasmacytic infiltrative inflammatory kidney disease [2]. PRL may be associated with some acute or chronic diseases (chronic pyelonephritis, Sjögren's syndrome, systemic erythematous lupus, or Epstein-Barr virus infection) [3].

PRL is a systemic disease manifesting initially in the kidneys, with poor outcome even if localized. It disseminates rapidly and mean survival is less than 1 year after diagnosis [4]. Renal involvement is seen in disseminated disease or in relapse. PRL is mostly of diffuse large B-cell lymphoma (DLBCL) type, while FL is exceptionally unusual, with scarce literature data.

Standardized PRL treatment is undefined. R-CHOP could be the elective treatment according to experience and some recently published studies [5,6]. Improved overall survival (OS) in FL with the addition of rituximab to conventional chemotherapy is well documented. High-grade FL and SUV were our major arguments for applying anthracyclines. We expected a better response rate (OS not improved) and reduced risk of FL transformation into DLBCL. The patient's transforming status was speculated. Cardiotoxicity (early/ delayed) and salvage therapy selection were the opposing arguments.

Address for Correspondence: Ivan PETKOVIC, M.D.

Niš University Clinical Center, Clinic of Oncology, Department of Hematological Malignancies, Niš, Serbia

Phone: +3810638020581 E-mail: ivan76.unsu@yahoo.com 


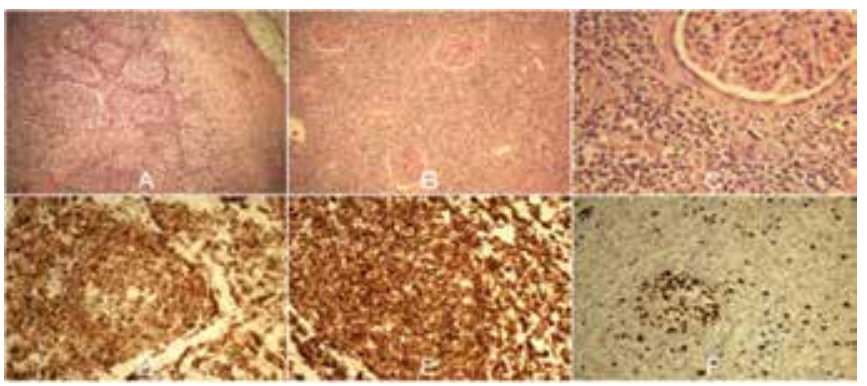

Figure 1. A, B, C) Representative H\&E stain photomicrographs of the kidney with grade 3A FL: A) typical follicular growth pattern in the kidney with FL, 40x; B) glomeruli immersed in diffuse lymphomatous mass of FL, 100x; C) glomerulus and renal diffuse lymphoma infiltration, 400x. D, E, F) Immunohistochemical staining representative for FL, 200x: D) diffuse positivity to CD20; E) intense staining of germinal center of neoplastic follicle with Bcl-2; F) Ki-67 proliferative activity (about20\%), 339x169 mm (72x72 DPI).

Long-term survivors occasionally have been reported after combined complete resection surgery/chemotherapy treatment, with longer disease-free survival and OS, but only one with kidney involvement $[1,7]$. The above-mentioned option is not the standard treatment. We advise that solid renal masses undergo biopsy and teamwork consideration (oncologists, radiologists, urologists) prior to eventual nephrectomy decisions.

In conclusion, solid renal lesions mimicking RCC may suggest underlying renal lymphoma infiltration. Although exceptionally uncommon, renal FL may be the pathological subtype. No standardized therapy exists in this specific situation. Rituximab with its documented survival benefit in FL is recommended to be included in the treatment regimen. The accompanying chemotherapy may depend on many variables, including ECOG performance status, patient age, FL grade, FLIPI index, and single-center decision.

\section{Conflict of Interest Statement}

The authors of this paper have no conflicts of interest, including specific financial interests, relationships, and/ or affiliations relevant to the subject matter or materials included.

Key Words: Primary renal lymphoma, Follicular lymphoma, Treatment option

Anahtar Sözcükler: Primer renal lenfoma, Foliküler lenfoma, Tedavi seçeneği

\section{References}

1. Okuno SH, Hoyer JD, Ristow K, Witzig TE. Primary renal non-Hodgkin's lymphoma. An unusual extranodal site. Cancer 1995; 75:2258-2261.

2. Omer HA, Hussein MR. Primary renal lymphoma. Nephrology (Carlton) 2007;12:314-315.

3. Stallone G, Infante B, Manno C, Campobasso N, Pannarale G, Schena FP. Primary renal lymphoma does exist: case report and review of the literature. J Nephrol 2000;13:367372.

4. Skarin A. Uncommon presentation of non-Hodgkin's lymphoma. Case 3. Primary renal lymphoma. J Clin Oncol 2003;21:564-569.

5. Belbaraka R, Elyoubi MB, Boutayeb S, Errihani H. Primary renal non-Hodgkin lymphoma: an unusual diagnosis for a renal mass. Indian J Cancer 2011;48:255-256.

6. Vazquez Alonso F, Sanchez Ramos C, Vicente Prados FJ, Pascual Geler M, Ruiz Carazo E, Becerra Massare P, Funes Padilla C, Rodriguez Herrera F, Cozar Olmo JM, Tallada Bunuel M. Primary renal lymphoma: report of three new cases and literature review. Arch Esp Urol 2009;62:461465.

7. Cupisti A, Riccioni R, Carulli G, Paoletti S, Tognetti A, Meola M, Francesca F, Barsotti G, Petrini M. Bilateral primary renal lymphoma treated by surgery and chemotherapy. Nephrol Dial Transplant 2004;19:1629-1633. 